\title{
Pleuroparenchymal fibroelastosis as a late complication of chemotherapy agents
}

\author{
To the Editor:
}

We identified six patients with clinical, radiographic and physiological features typical of pleuroparenchymal fibroelastosis (PPFE). In the six cases, PPFE may have been causally related to prior alkylating drugs used to treat malignacy, namely cyclophosphamide in five of the cases and carmustine (BCNU (1,3-bis-(2chloroethyl)-1-nitrosourea)) in one. Based on an extensive review of the literature, we suspect that similar cases may have already been reported in the past 4 decades but have not been recognised either as PPFE or as drug-induced in nature.

In 2004, Frankel et al. [1] described a then-new clinicopathologic entity, which they termed "idiopathic PPFE". The authors identified five patients who had a clinical presentation suggestive of chronic interstitial pneumonitis that did not fit any previously established category of idiopathic interstitial pneumonia. The patients were characterised by significant pleural involvement in the form of pleural thickening, which was more marked in both upper regions. In all five patients, surgical biopsy demonstrated prominent visceral pleural fibrosis with subpleural fibroelastosis and an abrupt transition between the fibrotic area and the near-normal adjoining lung. There was sparing of the underlying lung parenchyma at some depth from the pleural surface, and sparse fibroblast foci were present at the edge of the fibrotic area. Since that seminal report, several cases and small series have been reported that have demonstrated a strikingly similar clinical, radiological and pathological presentation, also characterised by difficult-to-treat pneumothoraces in a sizable fraction of affected patients.

In the updated American Thoracic Society/European Respiratory Society classification of idiopathic interstitial pneumonias [2], PPFE is included as a separate but well-defined category of rare entities. Little is known about the aetiology of PPFE and most cases are considered idiopathic. A few cases of PPFE have occurred amongst siblings [1,3]. PPFE has also been reported to be associated with a history of stem cell transplantation [4,5] or as a manifestation of chronic lung rejection in lung transplant recipients [5]. Recently, REDDY et al. [6] evaluated the pathologic and imaging results in 12 patients who fulfilled the established criteria for idiopathic PPFE. They hypothesised that there was a causal link between recurrent infections and a genetic or autoimmunity background; the hypotheses were not confirmed. The potential role of drug therapy as a cause for PPFE has not been discussed previously.

Here, we review the case records of six patients (three males), aged 15-37 years, from France, who presented with bi-apical pleural fibrosis encroaching the lung, with a clinical, radiographic and physiological presentation suggestive of PPFE. In four of them, the diagnosis of PPFE was confirmed by surgical biopsy of the lung and pleura; in the remaining two, a biopsy was not deemed necessary due to typical imaging findings and the attending risks. All six patients had a history of exposure to antineoplastic chemotherapy, which terminated 6 months to 10 years before symptomatic onset of their PPFE (table 1). Other factors aetiologically relevant to pleural thickening (e.g. exposure to asbestos, tuberculosis and other infections, coronary bypass graft surgery, trauma and haemothorax, connective tissue disease, renal failure, exposure to ergot alkaloids, and radiation therapy to the chest or breast) were absent. Three of our patients received total body or craniospinal irradiation. Table 1 shows the patients' history of malignancy, type of antineoplastic therapy and clinical characteristics. Remarkably, five of the patients had received cyclophosphamide and one had received other alkylating agents, including the nitrosourea BCNU, in the past.

Interpretation of the cases was complicated by underlying disease and the concomitant or sequential use of several chemotherapeutic agents, any of which can produce toxic manifestations. In support of our clinical impression, in a search for drugs as a cause for late pleural/pleuropulmonary toxicity, we found earlier cases of pleuropulmonary fibrosis that developed after exposure to the alkylating drugs BCNU or cyclophosphamide. In 1978, AlvarAdo et al. [7] studied two children who had received cyclophosphamide for the treatment of haematological malignancy. They presented with a peculiar pattern of "lung fibrosis" that predominated in the upper lobes and was thought to account for the reduced sagittal diameter of the chest.

For editorial comments see page 289. 


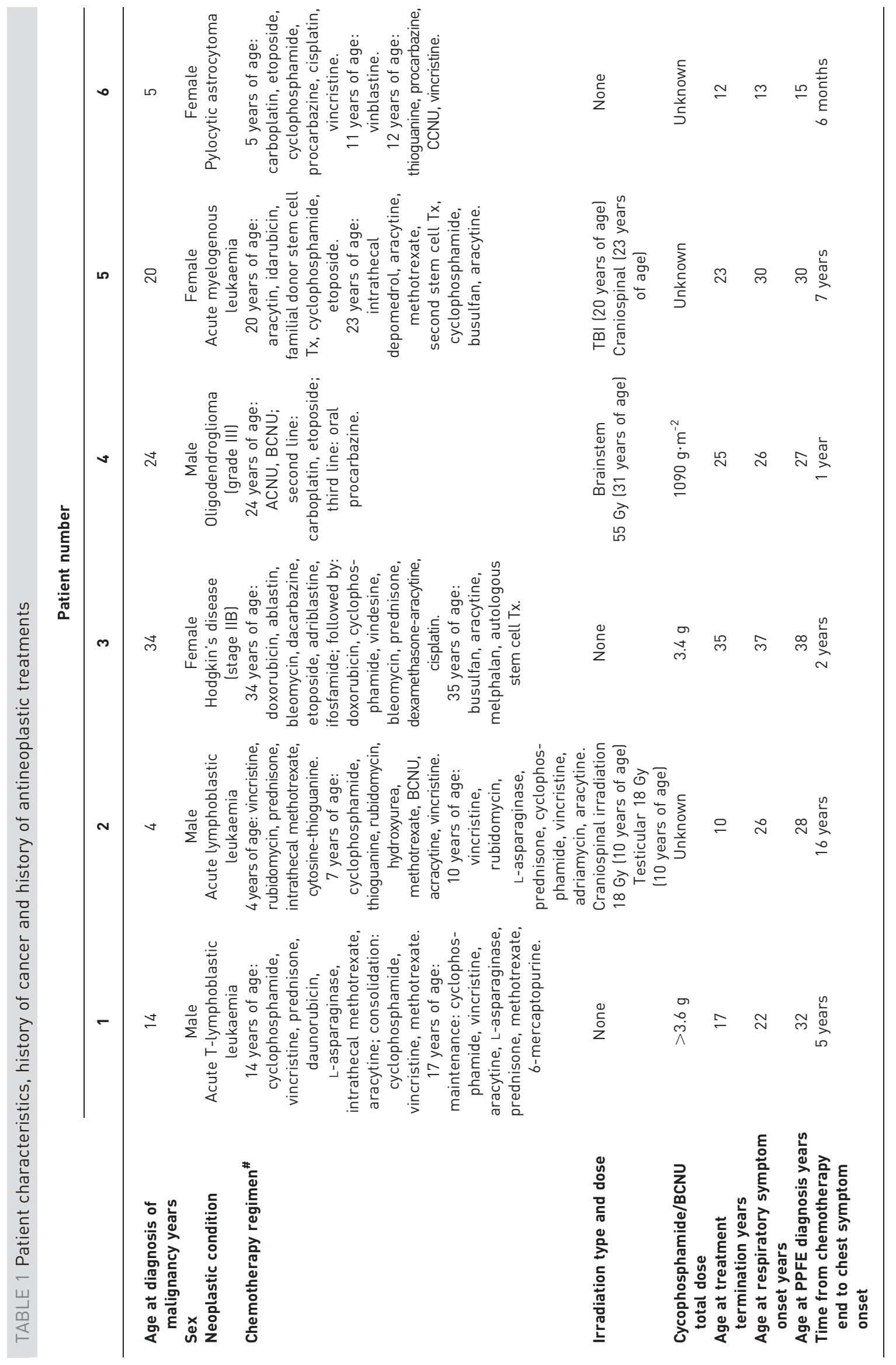




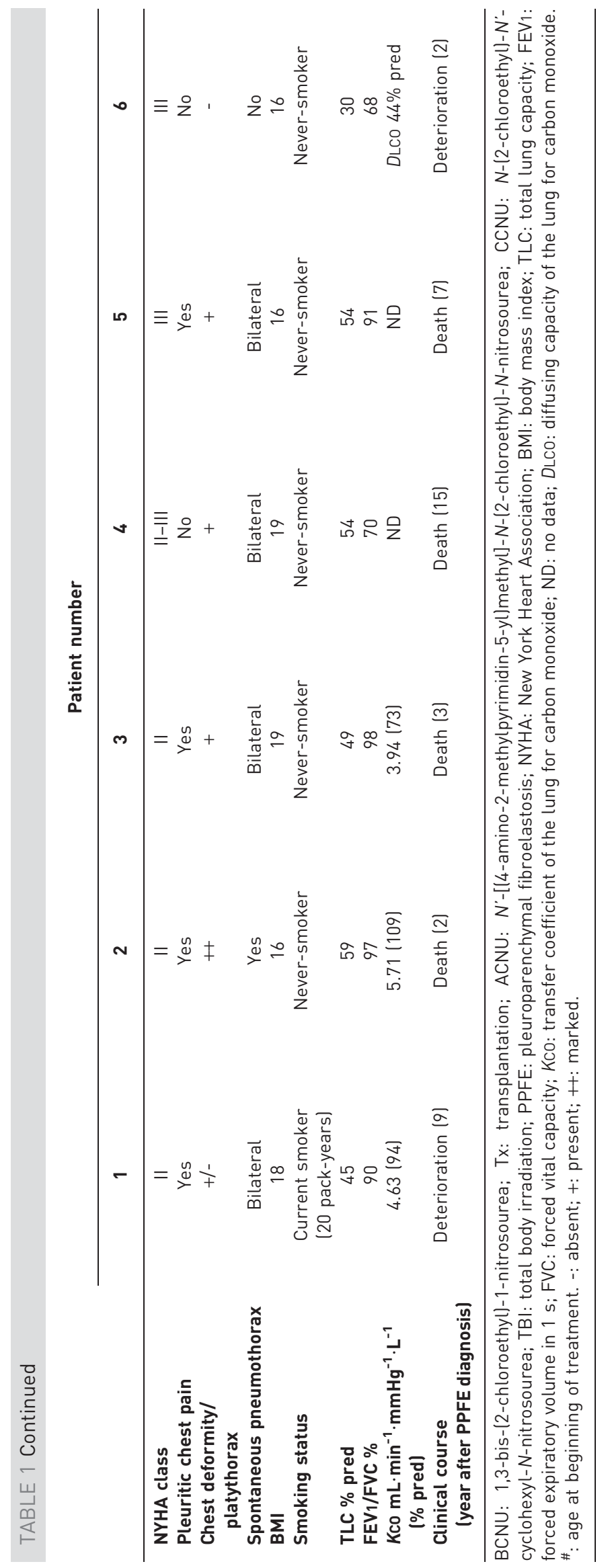


Chest deformity (also referred to as platythorax) increased during the progression of PPFE [8] and this finding was also present in five of our own patients. In a review of all lung toxicity cases suspected of being caused by cyclophosphamide over a 20-year period, MALIK et al. [9] identified two distinct patterns of cyclophosphamide-induced lung injury: 1) early onset pneumonitis that improved after cessation of the drug; and 2) late-onset pleuropulmonary fibrosis developing and progressing after cyclophosphamide was discontinued. Interestingly, most of the late-onset cases were clinically and radiologically similar to the recently described PPFE cases. Unfortunately, lung biopsies were not taken, and therefore the diagnosis of PPFE remain elusive. Furthermore, three of seven recently published definite PFFE cases [1, 10] also received cyclophosphamide but the possible causal role of the drug was not discussed. One further patient from the literature was described as being "immunosuppressed following a renal transplant for antineutrophil cytoplasmic antibody-positive glomerulonephritis" [6] and it is possible, if not likely, that he had been given cyclophosphamide as well.

Carmustine is a nitrosourea drug that is widely used to treat malignant brain tumours, Hodgkin's and nonHodgkin's lymphoma and multiple myeloma. In 1991, TAYLOR et al. [11] reported on six children who received carmustine for malignant brain tumours and later developed lung fibrosis. The authors noted that the upper lobes were predominantly affected [11, 12]. Five of their patients also exhibited pleural abnormalities on computed tomography (CT), including one with spontaneous pneumothorax: "a coarse linear strand lying predominantly in the upper lobes producing a subpleural band" [11]. A similar pattern was later reported in a patient treated with carmustine and cyclophosphamide [13].

In our study, the delay time between the end of chemotherapy and the first noticeable clinical symptom ranged 1-16 years. Such a lag in time is a potential pitfall in the identification of the offending agent. The onset of symptoms was generally insidious, with patients complaining of slowly progressive dyspnoea, nonproductive cough and pleuretic chest pain. Importantly, all patients had a normal chest radiograph prior to the onset of chemotherapy, and, in five of the patients, a chest radiograph taken immediately after termination of chemotherapy also appeared normal. The long delay mentioned previously is reminiscent of asbestos- and ergot-induced pleural involvement.

Two cases in our study (patients 3 and 5) had received an autologous or an allogenic hematopoietic stem cell transplantation as well as chemotherapy. VON DER THÜSEN et al. [4] recently described four bone marrow transplant recipients who went on to develop PPFE during follow-up. At least one of their patients had received cyclophosphamide. All four cases presented with subpleural fibrosis and pneumothorax on highresolution CT, with various grades of obliterative bronchiolitis and subpleural changes on pathology. Interestingly, one of their patients had received an autologous bone marrow transplant [4], as did patient 3 in our study. To our knowledge, bronchiolitis is almost exclusively associated with allogenic (not autologous) bone marrow transplantation. We feel it is reasonable to speculate that chemotherapy agents used for conditioning and/or dedicated treatment of haematological disease should be considered as a possible causative factor in the pathophysiology of PPFE.

In their study, REDDY et al. [6] suspected a link between PPFE and recurrent pulmonary infection. They pointed out that Aspergillus fumigatus may be associated with the disease. The search for an infectious disease, using bronchoalveolar lavage at the time of diagnosis, was negative in all six of our patients. However, during the course of their PPFE illness, Aspergillus infection was suspected in two patients and had complicated the course of the established fibrotic disease.

Four out of our six patients received corticosteroid therapy without any documented benefit.

The present study and previous reports of PPFE are consistent with the view that alkylating agents, specially cyclophosphamide and BCNU, are causally-related to PPFE, although a direct cytotoxic effect of the drug has yet to be proven. We believe that an accurate history of exposure to drugs is warranted in any PPFE to come.

0 @ERSpublications

Pleuroparenchymal fibroelastosis may be induced by exposure to alkylating agents http://ow.ly/vnuH5

Caroline Beynat-Mouterde ${ }^{1}$, Guillaume Beltramo ${ }^{1}$, Guillaume Lezmi ${ }^{2}$, Didier Pernet ${ }^{3}$, Clio Camus ${ }^{1}$, Annlyse Fanton ${ }^{1}$, Pascal Foucher $^{1}$, Vincent Cottin ${ }^{4}$ and Philippe Bonniaud ${ }^{1,5,6}$

${ }^{1}$ Service de Pneumologie et Soins Intensifs Respiratoires, CHU Le Bocage, Dijon, France. ${ }^{2}$ Service de Pneumologie Pédiatrique, Hôpital Necker-Enfants Malades, Paris, France. ${ }^{3}$ Service de Pneumologie, CHU Jean Minjoz, Besançon, France. ${ }^{4}$ Louis Pradel Hospital, Claude Bernard Lyon 1 University, Lyon, France. ${ }^{5}$ Faculté de Médecine et Pharmacie, Université de Bourgogne, Dijon, France. ${ }^{6}$ INSERM U866, Dijon, France. 
Correspondence: Philippe Bonniaud, Service de Pneumologie et Soins Intensifs Respiratoires, CHU Le Bocage, 21079 Dijon, France. E-mail: philippe.bonniaud@chu-dijon.fr

Received: Dec 102013 | Accepted after revision: March 262014 | First published online: May 22014

Conflict of interest: Disclosures can be found alongside the online version of this article at erj.ersjournals.com

\title{
References
}

1 Frankel SK, Cool CD, Lynch DA, et al. Idiopathic pleuroparenchymal fibroelastosis: description of a novel clinicopathologic entity. Chest 2004; 126: 2007-2013.

2 Travis WD, Costabel U, Hansell DM, et al. An official American Thoracic Society/European Respiratory Society statement: update of the international multidisciplinary classification of the idiopathic interstitial pneumonias. $A m$ J Respir Crit Care Med 2013; 188: 733-748.

3 Azoulay E, Paugam B, Heymann MF, et al. Familial extensive idiopathic bilateral pleural fibrosis. Eur Respir J 1999; 14: 971-973.

4 von der Thüsen JH, Hansell DM, Tominaga M, et al. Pleuroparenchymal fibroelastosis in patients with pulmonary disease secondary to bone marrow transplantation. Mod Pathol 2011; 24: 1633-1639.

5 Hirota T, Fujita M, Matsumoto T, et al. Pleuroparenchymal fibroelastosis as a manifestation of chronic lung rejection? Eur Respir J 2012; 41: 243-245.

6 Reddy TL, Tominaga M, Hansell DM, et al. Pleuroparenchymal fibroelastosis: a spectrum of histopathological and imaging phenotypes. Eur Respir J 2012; 40: 377-385.

7 Alvarado CS, Boat TF, Newman AJ. Late-onset pulmonary fibrosis and chest deformity in two children treated with cyclophosphamide. J Pediatr 1978; 92: 443-446.

8 Harada T, Yoshida Y, Kitasato Y, et al. The thoracic cage becomes flattened in the progression of pleuroparenchymal fibroelastosis. Eur Respir Rev 2014; 23: 263-266.

9 Malik SW, Myers JL, DeRemee RA, et al. Lung toxicity associated with cyclophosphamide use. Two distinct patterns. Am J Respir Crit Care Med 1996; 154: 1851-1856.

10 Becker CD, Gil J, Padilla ML. Idiopathic pleuroparenchymal fibroelastosis: an unrecognized or misdiagnosed entity? Mod Pathol 2008; 21: 784-787.

11 Taylor PM, O'Driscoll BR, Gattamaneni HR, et al. Chronic lung fibrosis following carmustine (BCNU) chemotherapy: radiological features. Clin Radiol 1991; 44: 299-301.

12 O'Driscoll BR, Hasleton PS, Taylor PM, et al. Active lung fibrosis up to 17 years after chemotherapy with carmustine (BCNU) in childhood. N Engl J Med 1990; 323: 378-382.

13 Parish JM, Muhm JR, Leslie KO. Upper lobe pulmonary fibrosis associated with high-dose chemotherapy containing BCNU for bone marrow transplantation. Mayo Clin Proc 2003; 78: 630-634.

\section{The effect of roflumilast in addition to LABA/LAMA/ICS treatment in COPD patients}

\author{
To the Editor:
}

Recently, roflumilast has been added as a therapeutic option for chronic obstructive pulmonary disease (COPD). Roflumilast is a selective phosphodiesterase type 4 (PDE4) inhibitor [1]. The main concern of the large-scale studies performed was that they did not assess the effect of roflumilast in addition to a combination product such as long-acting $\beta_{2}$-agonists (LABA) or long-acting muscarinic agents (LAMA) combined with inhaled corticosteroids (ICS) [2]. A novel technology called functional respiratory imaging (FRI) defines, in great detail, lung geometry and regional changes in airway volume and resistance [3-5]. By assessing changes close to the site of action of the intervention, the method is more sensitive (higher effect size) compared with standard pulmonary function tests (PFT) [3]. This implies that the mode of action of an intervention can be assessed in a small number of patients, while maintaining sufficient power to have statistically significant results $[3,4]$. We performed a study aimed at investigating the mode of action of roflumilast in COPD patients on top of triple therapy (LABA/LAMA/ICS), using PFT, exercise tolerance tests, patient-reported outcomes (PRO) and FRI. The hypothesis was that roflumilast provides a large benefit to a subset of COPD patients and that FRI can assist in phenotyping these responders. Ideally, a PFT parameter could be defined that could identify the responding phenotype.

A total of 41 COPD patients were included from January 2012 until October 2012 in two clinical centres. Patients were block randomised with a ratio of three treated to one placebo control subject, to eliminate any seasonal influences. In total, 30 patients were treated with roflumilast and 11 patients received placebo. The 